\title{
TWO-DIMENSIONAL DIFFUSION IN IRROTATIONAL CONVECTION
}

\section{Allan Paull}

This thesis concentrates on the steady two-dimensional Helmholtz vorticity equation which is linearised by Oseen's (1910) approximation as generalised by Burgers (1921). Under this approximation to the viscous flow equations, the fluid circulation behaves analogously to heat in that both diffuse down the gradients of their densities and are convected by a forced irrotational flow field. These linearisations can be used to determine solutions to flows past semi-infinite bodies in the far field, where the retention of a convection term is essential to describe the locally exponentially small vorticity (circulation area density).

In this thesis, flux functions and new analytical techniques are developed for diffusion of heat from point or distributed causes in forced irrotational convection fields. These functions and techniques are then used to develop solutions of the fourth order Oseen linearised flow equation. In addition, Green's functions appropriate for semiinfinite, boundary value problems for this fourth order equation are developed with the new techniques. Extensive analysis of the fourth order Oseen equation provides a firm foundation from which analytical solutions of the more complicated, fourth order equations for Burgers flow past a wedge or parabola may be obtained. Although this thesis does not provide exact analytical representations of stream functions induced in these more complicated convection fields, it does provide an insight into the particular problems of non-slip flows past a wedge and parabola.

The thesis is divided into two parts. Part I develops solutions of the second order diffusion-convection equation for heat (or circulation), while Part II addresses the fourth order linearised viscous flow problems. In each part solutions describing free or fundamental singularities are obtained first and then used in semi-infinite boundary value problems.

The point of view taken in Part $I$ is that the density function (temperature or vorticity) and the flux function provide alternative physical descriptions of diffusionconvection problems. The knowledge of one such function suffices, but the knowledge of both gives an enriched understanding. Single integral representations are given of the

Received 23 December 1987. Thesis submitted to University of Queensland, March 1987. Degree approved October 1987. Supervisor: Professor A.F. Pillow

Copyright Clearance Centre, Inc. Serial-fee code: 0004-9729/88 \$A2.00+0.00. 
flux function for diffusion of heat from the fundamental singularities of a point source and dipole in both uniform and stagnation-point convection fields. These flux functions are derived from their associated temperature fields by an integration of the generalised Cauchy-Riemann equations. These equations are modifications of the standard CauchyRiemann equations and include the effects of convection as well as diffusion.

Part I also develops new techniques for boundary value problems in which the temperature field is specified on a semi-infinite boundary in a uniform flow convection field. This new approach avoids the conventional use of Wiener-Hopf techniques. Its simplicity allows known results, which relate the temperature and flux function on a downstream boundary, to be simplified and generalised to include an arbitrarily orientated boundary. The Green's function which determines the temperature field from a convolution with the boundary temperature is obtained using these new techniques. This Green's function is then interpreted in terms of its simpler point singularities in the stagnation-point flow plane. An example in which the car radiator problem is solved is also included in order to display the use of Wiener-Hopf techniques.

In Part II the Splitting Theorem shows that the velocity induced in a forced uniform flow convection field can be written as a linear combination of the flux function and vorticity field which induced it, plus additional harmonic terms. The precise singular natures of these harmonic functions are obtained in terms of the singular nature of the vorticity field. The Splitting Theorem is used to determine the stream functions induced in a uniform flow by the fundamental singularities of Part $I$.

From Green's formula it is shown that the stream function induced in a uniform flow convection field which satisifes a specified velocity boundary condition can be obtained as a convolution between an appropriate Green's function and this specified velocity. The Green's functions suitable for semi-infinite boundaries which extend either upstream or downstream parallel to a uniform flow convection field are obtained. From Green's formula it is shown that these functions can be obtained from solutions of the fourth order adjoint Oseen equation which satisfy homogeneous boundary conditions. These solutions are interpreted as stream functions induced by distributions of circulation causes along the semi-infinite boundaries. These distributions are obtained by using the new techniques developed in Part I. Wiener-Hopf techniques are not required. It is shown that some terms of this distribution can be analytically continued into point singularities in the stagnation-point flow plane. This partial image system, together with three criteria, is used to define an alternative boundary value problem from which the remaining distribution of circulation singularities can be obtained. It is believed that these singularities and criteria provide a more fundamental approach to such problems.

In a particular example the stream function for non-slip, uniform flow past a semi- 
infinite flat plate is recovered anew analytically. The density of the circulation dipoles along this plate is a solution to the Miyagi-Bairstow integral equation.

Part II concludes with a chapter which explores the development of stream functions induced in three irrotational convection fields; (i) in area source, (ii) flow past a wedge and (iii) flow past a parabola. In particular, for the non-slip problems, the induced stream functions which have a velocity that balances the forced irrotational flow velocity along the natural boundaries of these convection fields are sought. An iterative technique, which is asymptotically correct for small wedge angles, is obtained for the non-slip flow past a wedge, and the isovorts, flux lines and streamlines for the non-slip flow past a parabola are also drawn. The integral equations that need to be solved for the fourth order Green's functions for the above boundaries and irrotational convection fields are also formulated.

Throughout this thesis computer plots of isovorts (isotherms), flux lines and streamlines are included. 